\title{
Proceeding
}

Supplementary Issue: Autumn Conferences of Sports Science. Costa Blanca Sports Science Events, 18-19 December 2020. Alicante, Spain.

\section{Virtual reality and functional recovery}

\author{
ALESSANDRO CAPRIOTTI $\triangle$, MAURO CASSOL, ARIO FEDERICI \\ Department of Biomolecular Sciences, University of Urbino "Carlo Bo", Italy
}

\begin{abstract}
While there are numerous traditional, non-three-dimensional software, proposed for the rehabilitation purpose, the number of software, based on virtual reality, used to aid the functional recovery of motor and cognitive skills, are drastically low in number even though, in recent years, their use is progressively expanding in all sectors, especially in the one of the health-motor. The benefits of these new technologies compared to traditional techniques alone in motor recovery following neurological traumas have been investigated. VR interventions provide enriched opportunities for integrating goal-oriented tasks and make repeated movements. The use of the headset is necessary to provide a more visually immersive environment (Weber, 2019). The results of functional recovery via immersive VR, have been shown to improve upper limb functions and self-care skills (Abant, 2019). The most interesting thing that emerged from these studies is a better involvement of those who carry out VR activities due to the full immersion of HMDs. It would be interesting in the future to carry out studies concerning motor activity in a VR environment in places with environmental limitations such as penitentiary facilities and retirement homes, also given the simplicity of learning that has emerged from the studies carried out.
\end{abstract}

Keywords: Immersive virtual reality (VR); Motor activity; Elder; Functional recovery.

\section{Cite this article as:}

Capriotti, A., Cassol, M., \& Federici, A. (2021). Virtual reality and functional recovery. Journal of Human Sport and Exercise, 16(2proc), S596-S600. doi:https://doi.org/10.14198/jhse.2021.16.Proc2.44

Corresponding author. Department of Biomolecular Sciences, University of Urbino "Carlo Bo", Italy.

E-mail: alessandro.capriotti@uniurb.it

Abstract submitted to: Autumn Conferences of Sports Science. Costa Blanca Sports Science Events, 18-19 December 2020. Alicante, Spain.

JOURNAL OF HUMAN SPORT \& EXERCISE ISSN 1988-5202

(c) Faculty of Education. University of Alicante

doi:10.14198/jhse.2021.16.Proc2.44 


\section{INTRODUCTION}

With the term Virtual Reality (VR) we often refer to all virtual environment, starting from classic $3 D$ applications that are used on regular screens. In recent years, new technologies have surfaced, such as Augmented Reality (AR) and Immersive Virtual Reality (IVR); we will begin by quickly introducing both to better understand their potential. We talk about "Augmented reality" when both the physical and digital world coexist on a sensory level (Microsoft, 2020) allowing the user to interact with both, without ever being confined in just one of the two. In the immersive virtual reality, the user experiences a digital environment while not being aware of what happens on the physical environment that surrounds them (Microsoft, 2020). Balance, the sense of sight, the hearing and the touch, all senses are stimulated (Meinhold, 2012).

\section{APPLICATION FIELDS}

The most interesting application can be seen in the medical field, where the IVR is often utilized in: surgeries, training doctors in delicate procedures (i.e., spinal cord or vertebral column medical operations); for motor and cognitive rehabilitation; for attention or memory disorder, or for autistic kids aid. There are apps that recreate an operating room, allowing students or surgeons to practice new techniques. By wearing the helmet, we can virtually become the surgeon and we can observe all the moves while he operates without actually doing the procedure while the brain records and becomes more aware (Maschuw, 2010). The advantages of IVR technologies are now broadly used in construction engineering studies (CEET), as they give the students the advantage to be able to interact between them, in a three-dimensional environment (3D). The intuitive nature of the subjects can be developed by also interacting with objects, messages and signals from the virtual world. Differently from the conventional learning approach, like for example the use of fixed images or bi-dimensional images (2D), the visual representation of the virtual reality makes it possible to integrate more degrees of freedom (DoF). Virtual reality has shown to be effective in providing a better comprehension and visual ability (Wang, 2018). Thanks to immersive VR one can plan a trip and try a virtual tour of the places and sightseeing before going on it. The real strength is the possibility to move around the rooms having a real perception of space. Immersive VR makes it possible to recreate historical sites and cultural place: they can be used for alternative learning methods that can motivate students to approach cultural things like museums, theatres and libraries (Eugene, 2019).

\section{MOTOR STUDIES}

The problems of the nervous system are very numerous and varied. According to a rough estimate by the World Health Organization dating back to 2017, there should be about a billion of people suffering from nervous system disorders. The causes are also the most diverse: infections, genetic mutations, trauma, nerve compressions, brain attacks (strokes), tumours, neurodegenerative diseases (such as Parkinson's and Alzheimer's disease). Through the application of new technologies, the world of motor re-education has also undergone a profound change. There is to consider the type of work that is carried out on these patients with the use of a state-of-the-art instrumentation compared to what has been used before. Very often these pathologies lead to a high social cost compared to rehabilitation costs and a loss of productivity on patients. In addition, the psychological impact on patients and their social environment, should not be underestimated, because many patients regress into depression. With high-tech equipment and using the 3D virtual reality headset, excellent results have been achieved in the field of rehabilitation of different deficits, resulted from an injury of the nervous system. Stroke is a common neurological problem and is one of the main causes of disability and death. In stroke patients, the mortality rate is about $30 \%$ and there is an increase in the morbidity rate after the occurrence of stroke. In addition, stroke is one of the main factors in the increase of burden of 
health care costs during adulthood. Upper limb paresis is the most common deficit after stroke. More than $80 \%$ of stroke survivors suffer from acute paresis of the upper limbs, and for half of them, paresis becomes chronic. Stroke can manifest itself as muscle tone disorders, weakness, loss of coordination and contractures. These impairments have a negative impact on the daily activities of the individual, including for example grasping, reaching and handling of objects. Immersive VR could create numerous realistic virtual environments, that could lead to new rehabilitation opportunities by providing a 360-degree interactive experience in a predefined environment where individuals are completely isolated from the outside world. In this environment, they can see their avatar and interact with objects. Virtual reality applications are the most advanced technology in use today and increase the individual sense of presence in the 3D environment (Ögün, 2019). Parkinson's disease is the second most common neurodegenerative disease. VR is used in the rehabilitation of neurological patients. VR technology emulates the real world in an environment where features are controlled, measurable, that is modifiable, allowing subjects to be isolated from non-natural research environments. The relation between VR and the human motor system is undoubtedly complex. However, it has been used to study the cognitive basis of complex movements such as navigation during locomotion and execution and observed interactions combined with functional magnetic resonance imaging (fMRI). VR-based systems are useful for studying motor behaviour. Some allow to evaluate the alteration and potentially treat rhythmic hand movements seen in Parkinson's disease and the elderly (Aria, 2012). There are recent theories about brain plasticity. Discoveries about the nervous system's ability to reconstruct cellular synapses as a result of interaction with enriched environments, have stimulated new research on memory rehabilitation. As a result, non-pharmacological, non-invasive cognitive rehabilitation (CR) interventions have gained increasing attention in recent years. Emerging VR applications today address the challenge of cognitive diagnosis and training of patients with mild cognitive decay (MCI) and dementia, focusing on navigation and orientation, facial recognition, cognitive function, and other instrumental activities of daily life (IADL). VR exposes patients with computer-generated cognitive disabilities in a virtual environment that provide a feeling of "presence" or "being there", so that the patient can interact in a multisensory way through near-naturalistic stimuli similar to real life. Using different perceptual aspects of psychophysics, mainly visual, tactile and kinesthetics perceptual sensations, virtual reality offers the possibility to perform activities, tasks and tests in a virtual environment adaptable to the various characteristics and needs of individual patients. A feature of VR, very useful for applications of Alzheimer's disease (AD), is the high level of interaction that can be achieved in a secure virtual environment (GarcíaBetances, 2015).

\section{RESULTS}

Performance Assessment of Self-Care Skills (PASS) scores were significantly higher in the VR group than in control $(p<0.05)$. FMUE and ARAT's clinically important minimum difference (MCID) scores were higher than the cut-off MCID scores described in the literature in the VR group, while FIM scores were lower than cut-off MCID scores. All scores in the control group were lower than cut-off scores. Immersive VR rehabilitation seemed to be effective in improving upper limb function and self-care skills but did not improve functional independence (Ögün, 2019). VR provides a significant improvement in both upper limb function and functional independence. Immersive VR has a significantly greater improvement in feature, independence and self-care testing than conventional therapies (Ögün, 2019). Based on WHO guidelines for endurance training, muscle activity during sessions occasionally reached the lower threshold for effective muscle activation. Muscle activation of the lower back corresponds to changes in the board with instability devices (for example, boards on gym balls) On the contrary, adapted VR workouts can provide better muscle strength, especially for the dorsal muscle chain. Differentiated muscle activation can be achieved in virtualized worlds, for example, requiring the user to spend more time in a pitch down position, thus moving 
the centre of gravity of the body to the pitch axis. But reaching the 30 minutes of daily cardiovascular activity recommended by the WHO requires dynamic movements instead of isometric. The gamification of physical exercise can help not only motivate physical activity, but also promote contact and social interaction. VR systems can offer to people a refreshing and enjoyable form of physical activity. In prevention and therapy, the quality of movement is a fundamental component of an effective resistance training for the benefit of health (Feodoroff, 2019). Virtual reality (VR) can simulate visuospatial environments where patients could practice in total safety and with results similar to classic treatments (Aria, 2012). Clinical scales showed improvements in motory functions and electrophysiological data showed an increased brain activation similar to healthy subjects, and brain imaging data showed the effect of motor imagery (MI) training and VR feedback, promoting plastic changes in targeted areas of the brain (Vourvopoulos, 2019). There was a small improvement in Fugl Meyer's average scores that did not achieve statistical significance from baseline. In addition, it seems that subjects are able to learn and use VR using only verbal signals as assistance. Patients seemed to appreciate the use of this system, stating that sessions passed quickly and were more fun. Several commented on the novelty of seeing their wall arm move normally within the system, particularly in positions typically limited by contracture (Weber, 2019).

\section{POSSIBLE PROBLEMS}

A study has shown that patients needed time to adapt to the VR system, and at least one session was needed for people to grasp the device and the user interface. During games where shoulder movement was required, 10 minutes sessions were exhausting and the movement quality was decreasing: for that reason, it was suggested to take a break, especially for repetitive upper limbs movements, based on patience's resistance. During rest, subjects can sit down without removing the VR device (Ögün, 2019). Added stress on both visual and vestibular system its more immersive but it can also cause proprioceptive signals that are less recepted by the user. In the future all body concept and completely immersive activities should focus on raise dynamic muscle activation, considering user's sensibility to cybersickness (Feodoroff, 2019) and motion sickness. In addition, all studies agree that there is a lack of work reference, and a lot of pilot studies were conducted on few subjects or without a control group; they also did not take into account self-learning and ecologically valid scenarios, through VR feedback (Vourvopoulos, 2019).

\section{CONCLUSIONS}

The advantages of VR technology in a rehabilitation field have been confirmed, as VR can help develop movement patterns (Vourvopoulos, 2019), decrease stress (Aram, 2017) and abstraction from an environment such as a hospital (Weber, 2019). In addition to the significant advantages from a motorrehabilitation point of view, conduct further studies on possible hypokinetic populations such as elderly people within RSA or in prisons, could be useful both from the point of view of training protocols, always through VR technology, to improve or preserve the residual skills of the subjects. We should increase the currently small number of reference publications in the motor field of VR technology, taking advantage of the critical issues that have emerged from previous studies. For example, trying to involve as many subjects as possible (Weber, 2019) for the study sample and for the control group that in some cases was absent (Vourvopoulos, 2019), taking advantage of the high involvement of the participants through immersive motor play activities (Feodoroff, 2019). In addition, it has been found that increasing the duration of sessions could lead to greater benefits. 


\section{REFERENCES}

Abant, B. I. (2019). Effect of Leap Motion-based 3D Immersive Virtual Reality Usage on Upper Extremity Function in Ischemic Stroke Patients. Arq. Neuro-Psiquiatr, vol.77 1678-4227. https://doi.org/10.1590/0004-282x20190129

Aram, K. D. (2017). Walking in fully immersive virtual environments: an evaluation of potential adverse effects in older adults and individuals with Parkinson's disease. Neuroeng Rehabil.

Aria, P. R.-G. (2012). Virtual Reality as a Tool for Evaluation of Repetitive Rhythmic Movements in the Elderly and Parkinson's Disease Patients. Plos One. https://doi.org/10.1371/journal.pone.0030021

Eugene, C. S.-T. (2019). Datasets from the evaluation of the adoption and use of digital technologies in China museums. Elsevier.

Feodoroff, B. K. (2019). Effects of Full Body Exergaming in Virtual Reality on Cardiovascular and Muscular Parameters: Cross-Sectional Experiment. JMIR serious games, 7(3), e12324. https://doi.org/10.2196/12324

García-Betances, R. I.-U. (2015). A succinct overview of virtual reality technology use in Alzheimer's disease. Frontiers in aging neuroscience, 7,80. https://doi.org/10.3389/fnagi.2015.00080

Maschuw, K. I. (2010). Chirurgisches Training am Simulator. Springer Link. https://doi.org/10.1007/s00104-009-1757-1

Meinhold, R. M. (2012). Virtual Reality. Salem Press Encyclopedia of Science.

Microsoft. (2020, Agosto 26). Che cos'è Realtà mista? Retrieved from Microsoft: https://docs.microsoft.com/it-it/windows/mixed-reality/discover/mixed-reality

Ögün, M. N. (2019). Effect of Leap Motion-based 3D Immersive Virtual Reality Usage on Upper Extremity Function in Ischemic Stroke Patients. Arquivos de neuro-psiquiatria, 77(10), 681-688. https://doi.org/10.1590/0004-282x20190129

Vourvopoulos, A. J. (2019). Efficacy and Brain Imaging Correlates of an Immersive Motor Imagery BClDriven VR System for Upper Limb Motor Rehabilitation: A Clinical Case Report. Frontiers in Human Neuroscience, 13: 244. https://doi.org/10.3389/fnhum.2019.00244

Wang, P. W.-L. (2018). A Critical Review of the Use of Virtual Reality in Construction Engineering Education and Training. Int $J$ Environ Res Public Health, 8;15(6):1204. https://doi.org/10.3390/ijerph15061204

Weber, L. N. (2019). Immersive virtual reality mirror therapy for upper limb recovery following stroke: A pilot study. Am J Phys Med Rehabil, 98(9): 783-788. https://doi.org/10.1097/PHM.0000000000001190

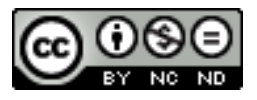

This work is licensed under a Attribution-NonCommercial-NoDerivatives 4.0 International (CC BY-NC-ND 4.0). 